\title{
Déchets de très faible activité : l'impact sur l'environnement
}

\author{
J.-C. BARESCUT*
}

(Manuscrit reçu le 20 novembre 1997, révisé le 23 février 1998, accepté le 8 mars 1998)

RÉSUMÉ Un des moyens de réduire l'impact d'un stockage de déchets est de limiter son contenu de telle sorte qu'après une durée raisonnablement courte, sa radioactivité ne dépasse pas significativement celle de son environnement. Pour que ce moyen soit pleinement efficace, il faut, entre autres, prouver que l'évolution spontanée du stockage et de son environnement ne va pas conduire localement à des taches d'activité supérieure à celle de la source d'origine. Cette concentration nécessiterait de toute évidence des migrations convergentes importantes. De telles migrations offriraient alors de nombreuses possibilités de dilution par mélange des radionucléides contaminants soit avec des isotopes stables de ces éléments soit avec des éléments chimiques similaires dont ils seraient ensuite difficilement séparables par des processus naturels. La limitation de la perturbation apportée au milieu naturel est une précaution élémentaire pour éviter que le système ne recèle des réserves énergétiques détoumables vers des processus de concentration. Les protections artificielles doivent être optimisées pour avoir un rôle bénéfique pendant la phase initiale de la vie d'un stockage sans qu'il y ait d'effets pervers pendant la phase * long terme».

ABSTRACT Environmental impact of low concentrated wastes.

A means to reduce the impact of a waste landfill is to limit its content so that after a reasonnably short time, its radioactivity does not significantly exceed the natural level. For this means to be effective, it must be demonstrated that the system will not, in the long run, spontaneously evolve towards local spots of higher concentration. This process would require considerable converging migrations that would offer many opportunities for mixing and thus be inconsistent with concentration. Moreover, concentration is an energy-demanding process that will be limited by the availability of energy, especially in chemical form. For these reasons, it is wise to avoid placing a landfill where energy is likely to be more readily available than on the average. It is also advisable to avoid artificial protections, if their future evolution is not fully understood, since they necessarily contain an excess of energy relative to the undisturbed natural state.

* Institut de protection et de sûreté nucléaire, BP. 6, 92265 Fontenay-aux-Roses, France 


\section{Introduction}

L'impact sur l'environnement dont il sera question dans cet article est celui de déchets TFA «ultimes» et en aucun cas celui des opérations intermédiaires ayant conduit à cet état. Cela exclut donc l'impact de tout entreposage intermédiaire et ne concerne que des déchets disposés dans des sites de stockage sans intention de reprise ultérieure.

En toute rigueur, il y a impact sur l'environnement dès lors qu'une modification est perceptible. La surexploitation de l'environnement par l'homme et les progrès des instruments de mesure font que cette situation a toutes les chances de se généraliser. Il n'est pas aisé de distinguer des modifications sans conséquences et des modifications contenant en germe des désagréments ultérieurs. Le débat autour du gaz carbonique et de l'effet de serre est particulièrement représentatif de questions de ce type. Seule une connaissance parfaite, et sans doute inaccessible, du fonctionnement de la nature permettrait de tolérer des modifications dont l'innocuité serait démontrée. En l'absence de cette connaissance, le principe de précaution doit être appliqué de façon raisonnable c'est-à-dire en privilégiant les stratégies ayant l'action la plus faible sur l'environnement.

\section{Définition des TFA}

Il n'existe pas encore de «norme» de ce qu'est un déchet «TFA». Une définition, compatible avec le principe de précaution pourrait être qu'un système de stockage de déchets ne mérite le label TFA qu'à trois conditions :

- sa radioactivité doit, dans un délai relativement court, se situer au voisinage du bruit de fond,

- il doit être écocompatible, c'est à dire non susceptible de générer, même localement, des flux de produits radioactifs supérieurs aux divers flux «similaires» traversant la biosphère,

- il ne doit pas être susceptible d'évoluer spontanément vers des concentrations supérieures à sa concentration initiale.

Compte tenu du fait que la radioactivité de longue période (U, Th, K et leurs descendants) de la géosphère se situe autour $\mathrm{du} \mathrm{Bq} / \mathrm{g}$, la première condition reviendrait à limiter à cet ordre de grandeur, le contenu moyen en éléments à vie longue des TFA.

La concentration en éléments à vie courte est moins critique car les nuisances associées peuvent être éliminées par des protections adéquates et n'ayant pas besoin d'être de longue durée. Toutefois, pour ne pas être amené à des protections aussi renforcées que celles des stockages du type du centre de stockage de l'Aube (CSA), 
la limite d'activité devrait être placée assez bas. Un chiffre de $100 \mathrm{~Bq} / \mathrm{g}$ est l'hypothèse la plus couramment avancée (sans que ce soit une valeur «officielle»).

La condition de non concentration est essentielle car en son absence, un stockage initialement TFA pourrait très bien se transformer en un stockage non-TFA.

\section{Impact sur l'environnement en cas de changement de configuration}

Des études (Brun-Yaba, 1995 ; Brun-Yaba, 1997) ont montré qu'un stockage de déchets TFA aurait un impact extrêmement limité dans sa configuration initiale. Cette étude se fonde sur un déchet «moyen» (qui n'est à ce stade qu'une hypothèse de travail) ayant une activité de l'ordre de $10 \mathrm{~Bq} / \mathrm{g}$ dont 0,2 en $\mathrm{Pu}$ et 1 à 3 pour ${ }^{234} \mathrm{U}$ et ${ }^{238} \mathrm{U}$. Les évaluations de doses qui en résultent son largement inférieures aux limites de dose applicables au «fonctionnement normal», même pour des modes de stockage d'une grande rusticité (tranchée sans aucune barrière artificielle et recouverte de terre) et pour des scénarii sortant du cadre du fonctionnement normal.

Ce résultat favorable est bien entendu lié à une hypothèse clé qui est la limitation de la concentration des radionucléides. Tant que le stockage évolue peu par rapport à son état initial, l'impact reste insignifiant. La question est de savoir si des processus mécaniques, physico-chimiques ou biologiques ne vont pas conduire avec le temps, à une nouvelle répartition de la radioactivité impliquant localement des concentrations de radioactivité très supérieures au chiffre initial.

\subsection{Limitations dues à la disponibilité d'énergie libre}

Avant de recenser les modes connus ou vraisemblables de concentration et de dispersion, il faut rappeler qu'il existe de très fortes contraintes thermodynamiques. La loi de croissance de l'entropie de l'univers a en effet pour conséquence qu'une augmentation locale de concentration, qui est une diminution d'entropie, est forcément compensée ailleurs, ce qui revient à dire que le sous-système se concentrant doit évacuer de l'entropie. Cette évacuation n'est possible que pour un système dissipatif ouvert, alimenté par un flux d'énergie de haute qualité (mécanique, thermique à haute température...) compensé par une dissipation d'énergie de basse qualité (thermique à basse température).

Pour augmenter la concentration d'un polluant (ou de tout autre élément) d'un facteur 10, le potentiel thermodynamique d'une mole de ce polluant doit être remonté de $R T \log (10)$ soit autour de 10000 joules d'enthalpie libre. Une telle quantité est d'autant moins facile à trouver que le polluant sera nécessairement en compétition avec son environnement pour la capter. 
Les phénomènes géologiques (hydrothermalisme, cristallisation fractionnée des magmas) sont capables de mettre en jeu de telles énergies et d'opérer des concentrations notables. Cependant si l'on se limite à des échelles de temps «humaines », une seule source d'énergie primaire domine largement toutes les autres : le couple rayonnement solaire/rayonnement terrestre. Cette source est tout à la fois le moteur des diverses circulations (atmosphère, rivières, courants marins) et la source d'énergie permettant à la biomasse de se construire.

En moyenne chaque $\mathrm{m}^{2}$ de haute atmosphère reçoit $10 \mathrm{GJ}$ par an sous forme de photons. La haute température (autour de 4000 degrés) de ces photons fait que cette énergie est presque totalement libre. Compte tenu des frottements internes de la machine «terre», la plus grande partie de cette énergie se dégrade rapidement et il n'en reste qu'une petite fraction qui se convertit en énergie mécanique ou en énergie chimique éventuellement utilisable pour des opérations de concentration de polluants.

Le stockage de l'énergie chimique se fait par l'intermédiaire de la construction de biomasse au rythme approximatif d'un $\mathrm{kg}$ par $\mathrm{m}^{2}$ et par an. Comme il s'agit essentiellement de composés de type glucose ou cellulose, cela représente la constitution annuelle d'un stock de $10 \mathrm{MJ}$ d'enthalpie libre, soit quelques pour mille de l'énergie atteignant le sol. Comme bien entendu, la biomasse formée va finir par se détruire (chaque niveau trophique détruit de la biomasse à son profit), seule une petite part du stock d'énergie chimique accumulé ainsi sera éventuellement disponible pour une concentration de polluants.

Un deuxième stock d'énergie chimique «minérale » réside dans le fort gradient de potentiel redox entre la couche superficielle du sol et les roches profondes, ce gradient résulte indirectement de l'activité biologique sans laquelle l'atmosphère serait dépourvue d'oxygène.

En énergie mécanique le bilan est plus difficile. L'énergie cinétique de l'atmosphère ( 10 tonnes d'air au dessus de chaque $\mathrm{m}^{2}$ de sol) est de l'ordre du MJ par $\mathrm{m}^{2}$. Il est difficile d'imaginer un processus permettant d'extraire (au profit d'opérations de concentration de polluants) plus de quelques pour cent de cette énergie par jour. De même, l'évaporation conduit à élever chaque année de plusieurs milliers de mètres $500 \mathrm{~kg}$ d'eau pour chaque $\mathrm{m}^{2}$ de sol, soit $25 \mathrm{MJ}$ dont une partie peut être interceptée par les plantes qui utilisent l'évaporation pour faire circuler leurs fluides internes.

On en arrive donc à la conclusion que l'énergie chimique ou mécanique disponible pour concentrer des polluants est, en moyenne, au mieux de l'ordre du $\mathrm{MJ}$ par an et par $\mathrm{m}^{2}$, soit autour du Joule par gramme de sol et par an, si l'on considère que l'essentiel des réactions se passe dans le mètre supérieur de sol. Comme il n'y a pas de raison de penser que les polluants capteront cette énergie beaucoup plus efficacement que les autres constituants du sol, cela signifie 
que les polluants, qui sont en général des noyaux plus lourds que les constituants habituels des sols, ne capteront que quelques dixièmes de Joules par grammes, soit quelques dizaines de joules par mole pour des polluants dont la masse atomique est autour de 100 .

Si l'on rapproche ce chiffre des 10000 joules mentionnées plus haut, on voit qu'en régime stationnaire, l'augmentation d'un facteur 10 de la concentration d'un polluant (initialement stable, c'est-à-dire ne disposant pas d'un stock d'énergie initiale) nécessitera autour de 1000 ans à condition que le mécanisme concentrateur soit thermodynamiquement quasi parfait (par exemple un cycle fermé faisant intervenir des extractants très sélectifs et non détruits dans l'opération) et qu'il n'y ait pas de superposition de mécanismes diluant s'y opposant.

La conclusion inéluctable est que la concentration spontanée sera un mécanisme extrêmement rare qui ne pourra avoir lieu que dans des zones spécifiques de la biosphère où l'énergie se concentre fortement (exemple : les grands cours d'eau transportent de très fortes densités d'énergie). De telles zones de «front » naturelles sont nécessairement assez rares car elles sont soumises depuis des temps "géologiques» aux mécanismes dissipateurs d'énergie. En revanche, les accumulations locales d'énergie dues à l'action humaine risquent d'être beaucoup plus fréquentes car elles n'ont pas été soumises depuis longtemps à ces mécanismes. Par exemple, toute structure artificielle mettant en contact des matériaux différents peut être considérée comme un réservoir d'énergie.

Ces considérations théoriques sont confirmées par de nombreux exemples. En particulier les minerais ne se créent spontanément que très difficilement (exception faite pour le guano, mais c'est justement parce que les oiseaux qui en sont à l'origine collectent l'énergie sur une grande surface et la relâchent sur une petite surface!) par des processus rapides. Enfin, les polluants «stabilisés» dont le potentiel initial est déjà très bas (comme certains plastiques) se détruisent très lentement.

La concentration éventuelle d'un polluant passe par au moins trois étapes au cours desquelles les contraintes thermodynamiques sont à considérer :

- l'extraction du polluant de son site d'origine,

- son transport,

- son dépôt avec concentration dans un nouveau site.

Sans apport d'énergie (à condition que le déchet soit bien stabilisé), la première phase est en général spontanée mais très lente. Une extraction rapide est cependant possible par des mécanismes d'origine biologique. La deuxième phase dépend de la mobilité de la phase porteuse (eau en général). C'est la dernière étape qui est sans doute la plus soumise aux contraintes thermodynamiques. En effet si le dépôt se fait dans un site relativement homogène, il se fera nécessairement 
n'importe où et conduira donc à une diminution de concentration. Pour que le dépôt se fasse en un point précis, il faut qu'il y ait un très fort gradient de conditions physico-chimiques et c'est le maintien de ce gradient qui sera coûteux en énergie.

En résumé, un déchet peut donc se concentrer dans quatre cas de figure :

- soit il est initialement mal stabilisé et possède ainsi un surplus d'énergie disponible lui permettant de faire évoluer certains de ses constituants vers un état de plus fort potentiel,

- soit lors de sa migration, le contaminant rencontre des conditions physicochimiques avec lesquelles il n'est pas en équilibre,

- soit il rencontre une source d'énergie mécanique suffisante (ruissellement, vent),

- soit enfin, il rencontre une source d'énergie chimique (vraisemblablement d'origine biologique).

Il faut noter que le premier cas de figure est normalement peu vraisemblable si les déchets sont inertés, les deuxième et troisième cas ont peu de chances d'être très efficaces car leur effet aura le plus grand mal à contrebalancer l'effet de dispersion lié à toute migration. C'est finalement le dernier effet qui peut être le plus difficile à évaluer tant les réactions biochimiques sont riches.

\subsection{Exemples de dispersion}

La dispersion aéro ou hydrodynamique est un mécanisme très efficace. Les écoulements naturels ne sont jamais totalement uniformes et chaque zone de convergence (exemple les confluents entre ruisseaux) est une occasion de mélange et donc de réduction des concentrations. Du fait de l'inhomogénéité du paysage (vallonnements, terrain plus ou moins perméable...), le phénomène pourrait même se produire pour des écoulements laminaires et donc a fortiori pour des écoulements turbulents. Il n'y a guère que pour des tronçons de cours d'eau particulièrement calmes et sans affluents latéraux qu'un transport non dispersif serait envisageable.

Au rang de la mécanique, il faut aussi rajouter la dispersion par les labours et, en conditions naturelles, la dispersion par les animaux fouisseurs (l'action des vers de terre représente en quelques années, un effet similaire à un labour). À échéance de plusieurs siècles, il ne faut pas oublier également les importations et exportations de matière organique. Même s'il est vraisemblable qu'une forte proportion de matière organique est recyclée dans un périmètre restreint, le peu qui sera exporté à des distances significatives se mélangera très fortement à l'environnement. 


\section{DÉCHETS TFA : L'IMPACT SUR L'ENVIRONNEMENT}

Pour un radionucléide transporté en solution, la dilution isotopique est un mécanisme d'une grande efficacité s'il existe dans la nature d'autres isotopes du même élément (ex. : I, Cs, Sr, Co, H, C...). Même sans isotopes concurrents, l'existence d'éléments chimiques à propriétés voisines est aussi une occasion de dispersion. Par exemple, les terres rares sont très difficiles à séparer les unes des autres au point que dans des conditions naturelles, leurs proportions relatives sont relativement proches dans tous les compartiments de l'environnement (océans, croûte terrestre, manteau et même les météorites). Dans ces conditions, une terre rare radioactive (ex. : le Ce) libérée dans l'environnement aura tendance à se mélanger le long de son parcours avec les autres terres rares. Le processus sera très lent car les terres rares sont peu solubles mais il se fera nécessairement dans le sens de la dilution.

Un cas plus courant est celui du Ca et du Sr qui ont des propriétés chimiques assez proches, au point que le $\mathrm{Sr}$ se substitue assez facilement au Ca dans la calcite. La solubilité de la calcite variant assez sensiblement dans l'environnement (effet de température et de pression partielle de $\mathrm{CO}^{2}$ notamment), le Sr coprécipitera avec le $\mathrm{Ca}$ lorsque les conditions chimiques locales le permettront ; il se mélangera avec le Ca si les eaux dissolvent la calcite.

Dans tous ces exemples, deux cas de figure sont possibles : si l'élément est peu soluble, il bougera peu et ne se concentrera donc pas ; s'il est soluble, sa migration déclenchera son mélange avec ses homologues. Dans les deux cas, il n'y a pas de possibilité d'un mécanisme concentrateur.

\subsection{Exemples de concentration}

Un mécanisme efficace de séparation mécanique est le tri granulométrique. Lorsque des particules sont transportées par un courant d'eau, tout ralentissement du courant se traduit par le dépôt des particules en commençant par les plus grosses et les plus lourdes et en finissant par les plus petites et les plus légères.

Dans ces conditions, les polluants fixés préférentiellement sur les particules les plus fines (celles qui possèdent la plus grande surface par unité de masse) peuvent être enrichis.

En théorie, de nombreux processus chimiques concentrateurs sont envisageables dès lors que le polluant, ou son porteur, se déplace dans un gradient quelconque. Les gradients d'acidité ou de potentiel redox peuvent agir dans ce sens (par exemple le fer III se réduit en fer II en s'infiltrant et précipite dans les horizons profonds du sol). De même, pour les espèces chimiques sujettes à la sorption, le passage d'un milieu grossier vers un milieu à forte capacité cationique (comme une argile) se traduit nécessairement par un front de concentration. 
L'évaporation est un mécanisme fortement concentrateur (ex : les marais salants). La concentration des eaux de lixiviation vers une mare évacuant son eau par évaporation pourrait ainsi donner naissance à terme à une accumulation locale.

Il est important de noter que la tendance naturelle étant à l'affaiblissement spontané de tous les gradients, l'existence de «fronts » à fort gradient suppose des mécanismes élévateurs de potentiel pour combattre les phénomènes de diffusion. Ainsi les forts gradients entre les eaux douces des fleuves et l'eau salée des mers (qui sont générateurs de précipitations des colloïdes) ne sont possibles que par l'arrivée continue d'eau douce, celle-ci étant en dernière analyse le résultat de la remontée de potentiel exercée sur l'eau par le rayonnement solaire en l'évaporant. De même, les gradients verticaux au voisinage de la surface du sol sont dus au rayonnement solaire. Tous ces phénomènes sont fortement consommateurs d'énergie ce qui ramène à la limitation citée plus haut : la concentration d'éléments chimiques très dilués est limitée par la disponibilité d'énergie.

Il est connu que certains polluants organiques peuvent être bioamplifiés (c'està-dire que la concentration croit d'un niveau trophique au suivant). Les pesticides en particulier peuvent s'accumuler dans les animaux riches en graisse situés en bout de chaîne. Pire encore, la concentration peut s'accroître lorsque ces animaux consomment leur graisse en jeûnant.

Il y a moins d'exemples connus pour les polluants «minéraux ». L'algue laminaria digitata peut cependant accumuler jusqu'à $12 \mathrm{~g} \mathrm{~d}$ 'iode par $\mathrm{kg}$ de matière sèche. Le Tc semble pour sa part avoir un très fort facteur de concentration dans les algues (30000 à comparer à seulement $50 \mathrm{l} / \mathrm{kg}$ pour le Cs). Enfin, des cas de plantes accumulant des métaux lourds dans leurs feuilles sont également connus.

La concentration «minérale » n'est pas très surprenante car elle est associée à des mécanismes vitaux pour les plantes. Celles-ci puisent des éléments traces dans l'eau interstitielle du sol et les concentrent ensuite dans leurs tissus en évaporant l'eau. Les plantes ne se contentent d'ailleurs pas de pomper passivement une eau en équilibre avec le sol ; elles, ou leur symbiotes, émettent aussi des protons et des produits complexants (exemple les sidérophores qui sont d'excellent complexants du fer) qui permettent de solubiliser davantage certains éléments et de les attirer vers les racines (puisque tout se passe comme si la concentration était nulle). Ce type de mécanisme biogéochimique conduit finalement la biosphère à «modeler » la composition du sol sur lequel elle repose. La sélectivité imparfaite de ces complexants est peut-être une des raisons pour laquelle des polluants indésirables (métaux lourds en particulier) arrivent à pénétrer dans la plante. 
La bioamplification est cependant un phénomène limité car la disparition du prédateur situé en bout de chaîne s'accompagne nécessairement d'une remise en circulation des stocks accumulés.

\section{Le retour d'expérience en radioécologie}

C'est de la radioécologie que peuvent venir les enseignements majeurs sur l'évolution à long terme des stockages de surface. Le comportement dans l'environnement des retombées de Tchernobyl, de certains rejets massifs (rivière Techa dans l'Oural) ou plus modestes peuvent permettre de voir à l'œuvre certains des mécanismes mentionnés plus haut.
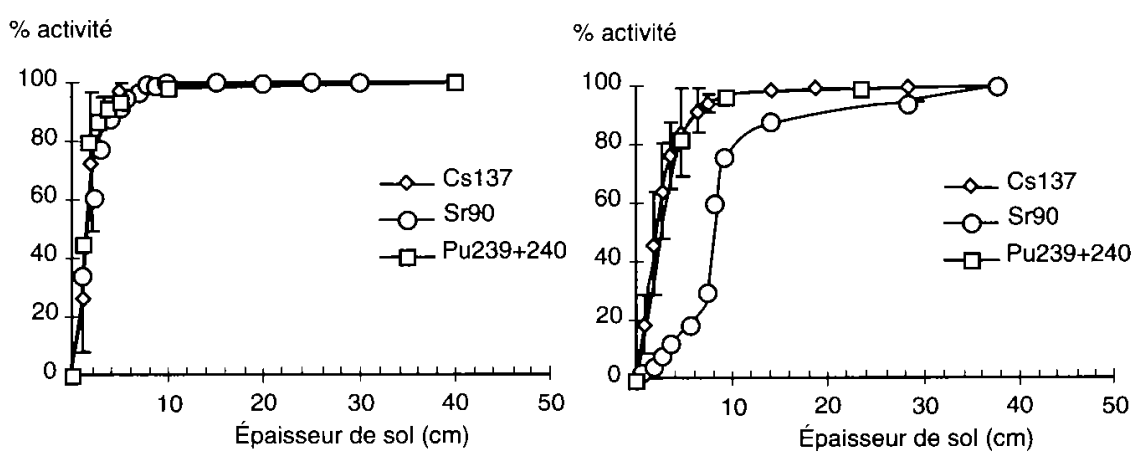

Fig. 1. - Répartition d'activité dans des podzols situés à $10 \mathrm{~km}$ (gauche) et à $150 \mathrm{~km}$ (droite) de la centrale de Tchernobyl.

Distribution of activities in podzols at $10 \mathrm{~km}$ (left) and $150 \mathrm{~km}$ (right) from Tchernobyl power plant.

\subsection{Les migrations verticales du Cs, Sr et Pu}

10 ans après l'accident de Tchernobyl, on peut constater (Fig. 1 selon Besnus, 1996) que la répartition de ces radioéléments reste concentrée dans les 10 premiers centimètres de sol, ce qui est la preuve d'une mobilité particulièrement faible. En particulier, à $150 \mathrm{~km}$ de la centrale, là où les retombées ne sont pas dominées par de grosses particules, le plus mobile de ces éléments (le Sr) atteint à peine une profondeur de $10 \mathrm{~cm}$.

On peut faire le même type de constatation en France. La figure 2 (Maubert, 1997) montre qu'en 10 ans, le profil vertical de ${ }^{137} \mathrm{Cs}$ n'a quasiment pas évolué dans un sol de la région du Haut-Var. 
Profil de radioactivité dans un sol forestier du Boréon

$\mathrm{Bq} / \mathrm{g}$ sec au 4/7/95

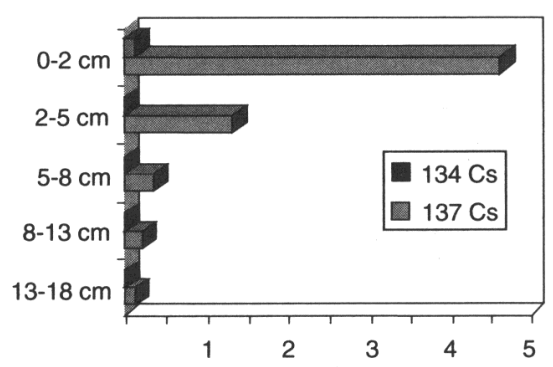

Profil de radioactivité dans un sol forestier du Boréon $\mathrm{Bq} / \mathrm{g}$ sec au 20/05/95

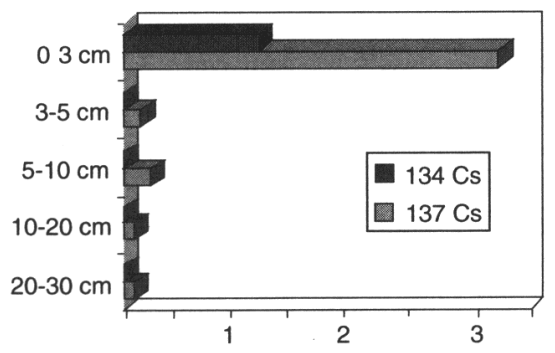

Fig. 2. - Comparaison entre des profils de radioactivité de sols forestiers du Boréon entre mai 1986 et juillet 1995.

Comparison between vertical profiles of soil radioactivity in the forest of Boreon between May 1986 and July 1995.

On peut donc tenir pour acquis que la migration verticale des radioéléments est un phénomène extrêmement limité à échelle de quelques dizaines d'années.

\subsection{Le «nettoyage» des zones continentales}

Les circulations d'eau dans la couche superficielle du sol sont essentiellement verticales à l'exception notable des circulations par ruissellement. Cette circulation par ruissellement peut être appréhendée par le suivi des sorties de radioéléments aux exutoires des bassins versants et dans les grandes rivières.

Il n'y a malheureusement que peu de bassins versants équipés correctement pour un tel suivi. Les informations ne sont donc que fragmentaires et liées à l'accident de Tchernobyl.

Dans le sud-est de la France (Mercantour), le vallon du Cavalet qui est un petit bassin versant de 2,6 km², a été étudié de façon fine (Maubert, 1997). À partir de 26 prélèvements, il a pu être établi que la moyenne du dépôt surfacique de Cs était de $31000 \mathrm{~Bq} / \mathrm{m}^{2}$ (valeur «normale» dans cette région) avec des fluctuations assez importantes.

L'information clé est que, à la sortie du bassin, l'eau contient actuellement $3 \mathrm{~Bq} / \mathrm{m}^{3}$ de ${ }^{137} \mathrm{Cs}$. Le ruissellement étant certainement inférieur à $1 \mathrm{~m}$ d'eau par an, cela signifie que la perte annuelle actuelle de Cs du bassin n'est que de $10^{-4}$ de son contenu. Immédiatement après l'accident, la concentration de l'eau n'était que de $30 \mathrm{~Bq} / \mathrm{m}^{3}$, ce qui correspondait à une perte de $10^{-3}$ par an. 
On peut en tirer deux conclusions :

- d'une part la contamination est fortement fixée dans le bassin,

- d'autre part, cette fixation est de plus en plus efficace puisque la concentration de l'eau a baissé d'un facteur 10 sans que le Cs ait bougé significativement.

\section{TABLEAU 1}

Évolution de la radioactivité des eaux de rivière.

Evolution over time of river water radioactivity.

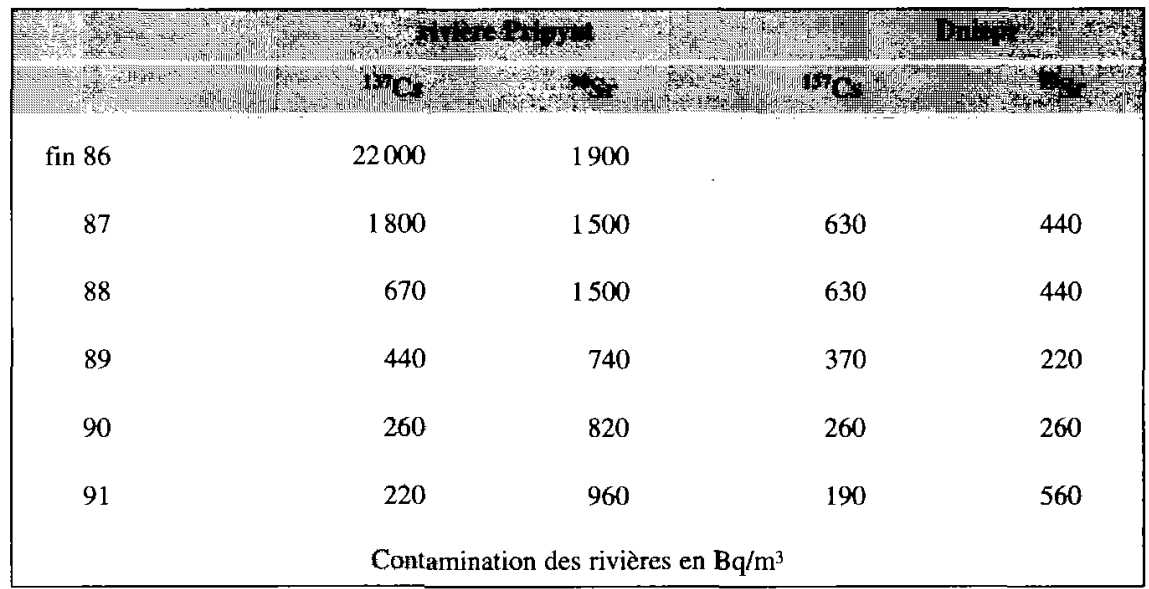

Le Sr peut difficilement être étudié en France car les retombées de Sr ont été beaucoup plus faibles que celles de Cs. On dispose cependant de données (Tab. 1 extrait de (Aarkrog, 1994)) particulièrement instructives sur la contamination des eaux de la rivière Pripyat et du Dniepr (qui drainent le voisinage de Tchernobyl). Un bilan similaire à celui fait pour le vallon du Cavalet montre que la perte par entraînement par les eaux a êté insignifiante.

La retombée de Cs dans la zone drainée par la Pripyat était de l'ordre de $1000 \mathrm{kBq} / \mathrm{m}^{2}$ soit à peu près 100 fois le chiffre du Sud-est de la France et la teneur en Cs des eaux est aussi de 100 fois celle des eaux du Cavalet.

Le Sr apparaît comme plus mobile que le Cs puisque pour des retombées en gros 10 fois plus faibles, l'eau est 5 fois plus contaminée en $\mathrm{Sr}$ qu'en Cs. Cela signifie sans doute que le Sr s'évacuera 50 fois plus vite que le Cs, ce qui reste néanmoins un phénomène très lent et négligeable devant la décroissance radioactive du Sr. 
Tous ces exemples montrent que les $\mathrm{Cs}, \mathrm{Sr}$ et $\mathrm{Pu}$ sont quasiment insensibles à l'entraînement par les eaux dans des conditions de ruissellement «normales ».

\subsection{L'action des forts ruissellements}

Dans les zones à fort ruissellement (ce qui ne devrait pas être le cas d'un stockage), des phénomènes notables de concentration sont possibles. Le point connu de France le plus contaminé par les retombées de Tchernobyl (314000 Bq/kg près d'Isola) est un replat au bas d'un petit bassin versant de montagne.

Le phénomène n'est pas totalement compris. L'effet de convergence des eaux vers une zone stagnante est sans doute en partie responsable mais l'on peut aussi avancer l'hypothèse que des particules fines de terrain ont été arrachées et se sont ensuite déposées dans la zone de ralentissement du courant.

Ce qui peut rendre le phénomène concentrateur, c'est que la contamination des particules est fonction de leur taille. Le Cs s'accumule prioritairement sur les constituants du sol ayant la plus grande surface par unité de masse.

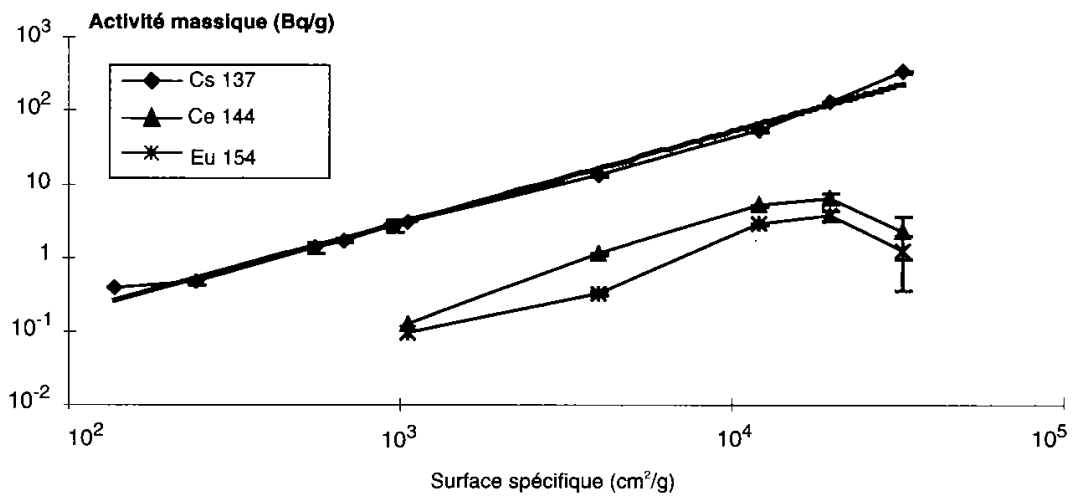

Fig. 3. - Répartition des activités massiques du ${ }^{137} \mathrm{Cs}$ et des ${ }^{144} \mathrm{Ce}$ et ${ }^{154} \mathrm{Eu}$ en fonction de la taille des particules de sols situés dans la zone des $10 \mathrm{~km}$ autour de la centrale de Tchernobyl.

Distribution of massic activities of ${ }^{137} \mathrm{Cs},{ }^{144} \mathrm{Ce},{ }^{154} \mathrm{Eu}$ according to the size of soil particles in the area $10 \mathrm{~km}$ around the Tchernobyl power plant.

Cet effet est confirmé par des mesures faites sur des échantillons de terrain contaminés de la zone d'influence de Tchernobyl (Fig. 3 selon Peres, 1996). Une analyse après tri granulométrique a mis en évidence que, pour un échantillon dont l'activité était de $7 \mathrm{~Bq} / \mathrm{g}$, la fraction de taille micronique contenait $60 \%$ de l'activité due au Cs et avait une activité massique 30 fois supérieure à la moyenne de l'échantillon. 


\subsection{L'action éolienne}

Le transport par voie aérienne est aussi un puissant mode de séparation des poussières selon leur taille puisque les poussières grossières retombent très vite et les poussières fines peuvent parcourir des distances importantes (y compris traverser les océans). Ce phénomène peut être gênant du point de vue de la radioprotection (cas du chantier traversant un site de déchets) mais est totalement négligeable du point de vue de l'environnement. À la différence de ce qui peut se passer pour la sédimentation en rivière, il est impossible que les poussières fines retombent toutes au même endroit.

La remise en suspension est donc très nettement un phénomène dispersif et non un phénomène concentrateur. En ce sens ce serait un phénomène plutôt favorable pour effacer à terme les restes d'un stockage TFA oublié. Cependant, au rythme de quelques grammes échangés chaque année pour chaque $\mathrm{m}^{2}$, le phénomène nécessite des durées «géologiques». Le retour d'expérience de l'accident de Palomarès montre que malgré des conditions climatiques favorables à la remise en suspension, le stock de plutonium restant n'évolue quasiment pas.

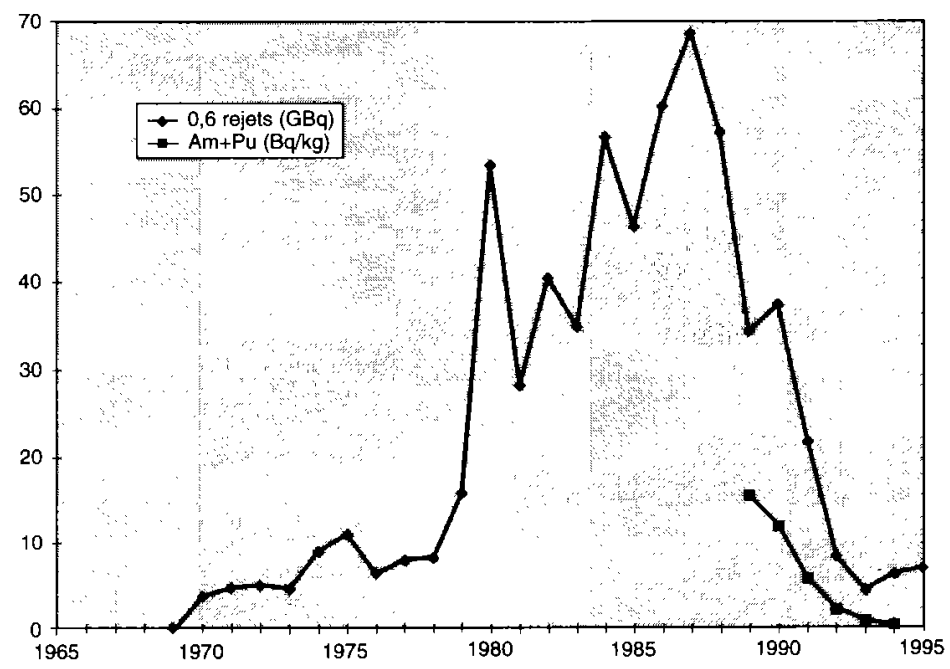

Fig. 4. - Rejets dans le Rhône de l'usine de retraitement de Marcoule et contamination des sédiments du Rhône à Vallabrègues.

Discharge of the Marcoule reprocessing plant in the Rhone river compared to contamination of sediment in Vallabrègues. 


\subsection{Les grands cours d'eau}

Les grands fleuves comme le Rhône sont des voies de transport particulièrement rapides. On peut vérifier (Fig. 4 selon Lambrechts) que deux ans après le pic de rejets d'émetteurs alpha (l'U qui représente $40 \%$ des émetteurs alpha a été exclu du décompte) de l'usine de Marcoule, la concentration ( $\mathrm{Pu}+\mathrm{Am}$ ) des sédiments du Rhône est déjà décroissante. Le Pu étant attribuable aux rejets de l'usine par sa signature isotopique, il est donc clair que le déphasage des pics est au plus de 2 ans (le pic dans les sédiments ne peut évidemment avoir lieu avant sa cause). Si la dispersion avait été plus faible, il y aurait eu un déphasage bien plus grand puisque le $\mathrm{Pu}$ aurait continué à s'accumuler même avec des apports nouveaux en décroissance. Les mécanismes de dispersion, que ce soit l'entraînement par les eaux ou le piégeage dans des sédiments plus profonds, l'emportent largement même sur une source relativement intense. A fortiori, l'arrêt des apports nouveaux entraînera une décroissance encore plus rapide de la concentration des sédiments superficiels et donc de la biomasse en contact avec ces sédiments.

La concentration des eaux du Rhône n’étant pas encore suivie en permanence, il est encore trop tôt pour déterminer quelle proportion du Pu manquant dans les sédiments de surface est charriée vers la mer.

\section{La biodisponibilité}

D'un point de vue «protection» de l'environnement, ce sont les compartiments «vivants» de l'environnement qui sont les plus importants. En plus des migrations «géographiques », il faut donc aussi étudier comment se font les répartitions de radioéléments entre ces compartiments vivants et le reste.

En moyenne sur toute la France, on peut constater que la contamination des productions agricoles a rapidement décru après Tchernobyl. La contamination du lait de vache par exemple a été divisée par 100 en 10 ans (Fig. 5). Sachant que les migrations horizontales et verticales ainsi que la décroissance radioactive sont faibles à cette échelle de temps, la seule explication possible à cette baisse est une nouvelle répartition des radioéléments entre un stock «biodisponible» (la biomasse et la fraction de sol avec laquelle il y a de forts échanges) et un stock peu disponible, difficilement lixiviable ( $c f$. la diminution du taux d'extraction par le drainage) et difficilement transférable par les racines des plantes. La seule exception notable est le cas des champignons qui pourrait s'expliquer par le fait que les écosystèmes forestiers sont beaucoup plus «clos» que les écosystèmes agricoles soumis à de fortes importations (engrais) et exportations (produits agricoles). Le système forestier recycle vraisemblablement de façon efficace et atteint rapidement un régime d'équilibre caractérisé par de faibles pertes.

Le même phénomène est visible dans les poissons d'eaux douces avec un facteur 100 de décroissance en 10 ans (Fig. 6). 


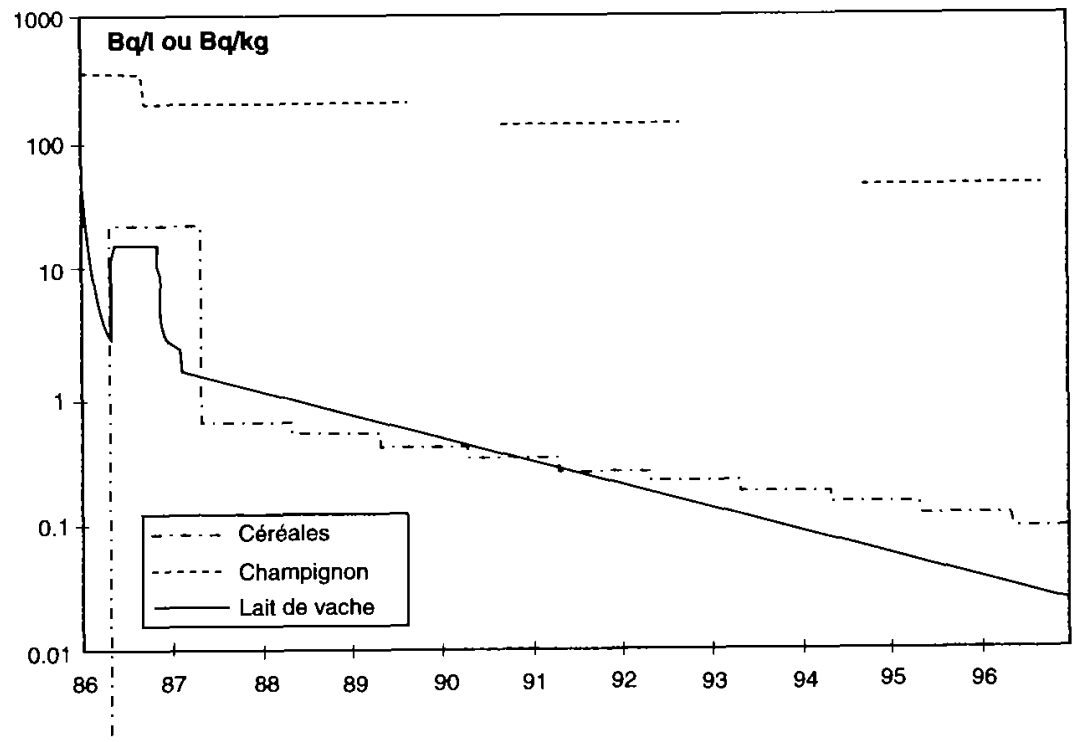

Fig. 5. - Evolution de la contamination moyenne (sur la France) de productions types de la biosphère.

Evolution over time of the mean contamination of typical biological products in France.

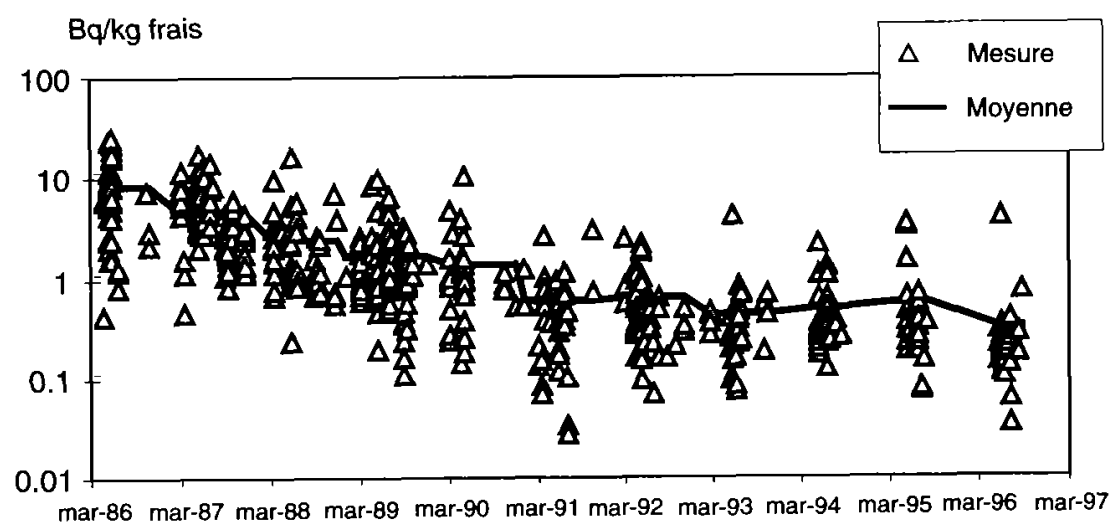

Fig. 6. - Concentration en ${ }^{137} \mathrm{Cs}$ des poissons d'eau douce.

${ }^{137}$ Cs contamination of fresh water fish. 


\section{Retour sur la compétition entre la dispersion et la concentration}

L'expérience montre que de grandes surfaces contaminées sont susceptibles d'évoluer en provoquant quelques points très localisés et très minoritaires à concentration plus élevée. Une telle situation est normale puisque les processus de dispersion sont de moins en moins actifs lorsque les dimensions augmentent (pour un écart donné de concentrations, le gradient qui commande les échanges est inversement proportionnel à la taille). Les mécanismes dispersifs étant faibles, il est alors possible que quelques fluctuations locales se développent et donnent lieu aux quelques cas observés de concentration.

En revanche si l'on part d'une anomalie de petites dimensions (un stockage) dans un environnement non perturbé, les gradients dispersifs seront nécessairement beaucoup plus intenses et les modes de dispersion l'emporteront sur les quelques modes de concentration à l'ouvre. Il est tout à fait invraisemblable que cela puisse conduire à un point chaud encore plus concentré que le stockage initial.

Ce raisonnement s'applique aussi bien à un polluant «nu » qu'à un polluant transporté par une particule solide (comme cela a été évoqué plus haut), un colloïde ou enfin un complexant organique.

\section{Conclusion}

Une caractéristique essentielle que doit posséder un stockage de déchets pour être considéré comme TFA est de ne contenir que très peu d'éléments à vie longue. Deux scénarii d'évolution sont alors possibles. Si les migrations sont faibles, la décroissance radioactive contribuera à faire diminuer l'impact avec le temps; si celui-ci est initialement acceptable, il le sera a fortiori dans l'avenir. En revanche, si les migrations sont significatives, cela conduira à favoriser les mécanismes dispersifs. Conjugué avec la décroissance radioactive, cela effacera encore plus les traces du stockage.

Un point important est qu'au-delà des quelques dizaines d'années initiales pendant lesquelles les éléments à vie courte sont dominants, l'existence de protections renforcées, dont la pérennité est nécessairement limitée (bétons, colis, barrières argileuses) n'apporte pas de meilleure garantie en termes de sûreté. Bien au contraire, l'ajout de matériaux étrangers en grande quantité augmente, relativement à un site "vierge », l'énergie libre localement disponible. Compte tenu des limites des méthodes de modélisation, il sera difficile de garantir que ce surplus d'énergie ne peut pas intervenir dans des processus physico-chimiques concentrateurs. Mieux vaut dans ces conditions éviter de se placer dans une situation 
pouvant poser des problèmes. Pour protéger les travailleurs et les voisins contemporains de la phase, les méthodes les moins «perturbantes» comme par exemple l'utilisation de matériaux «locaux» et la mise en place de servitudes sont à privilégier.

\section{RÉFÉRENCES}

Aarkrog A. (1994) Sources to environmental radioactive contamination in the former USSR. Radiation protection-71, European Commission.

Besnus F., Peres J.-M., Guillou P. (1996) Contamination characteristics of podzols affected by the Chemobyl accident. Dans : First international conference of the European commission, Belarus, Russian federation and Ukraine. Minsk. Rapport EUR 16544 EN.

Brun-Yaba Ch., Peres J.-M., Besnus F. (1995) Environmental impact of a very low waste specific landfill Dans : Troisième conférence de l'Oural. Zarechny.

Brun-Yaba Ch., Peres J.-M. et al. (1997) Impact radiologique d'une décharge spécialisée de déchets de très faible activité Rapport EUR n 17646 FR.

Foulquier L., Baudin Y. (1993) Le 137Cs dans les poissons : retour d'expérience après l'accident de Tchernobyl. Journal of radioecology, 1, 29-38.qq

Lambrechts A., communication personnelle.

Maubert H. et al. (1997) Note sur les valeurs de radioactivité mesurées dans le haut bassin du Var. Rapport IPSN/DPRE 97-04.

Peres J.-M., Besnus F. (1996) Contamination of surfaces by resuspended material. Final report of ECP1 Project. Rapport EUR n 16527 EN. 